PROCEEDINGS OF THE

AMERICAN MATHEMATICAL SOCIETY

Volume 133, Number 7 , Pages 1929-1933

S 0002-9939(05)07816-0

Article electronically published on January 31, 2005

\title{
DECOMPOSABLE FORM EQUATIONS WITHOUT THE FINITENESS PROPERTY
}

\author{
ZHIHUA CHEN AND MIN RU
}

(Communicated by Wen-Ching Winnie Li)

\begin{abstract}
Let $K$ be a finitely generated (but not necessarily algebraic) extension field of $\mathbb{Q}$. Let $F(\mathbf{X})=F\left(X_{1}, \ldots, X_{m}\right)$ be a form (homogeneous polynomial) in $m \geq 2$ variables with coefficients in $K$, and suppose that $F$ is decomposable (i.e., that it factorizes into linear factors over some finite extension of $K)$. We say that $F$ has the finiteness property over $K$ if for every $b \in K^{*}$ (here $K^{*}$ denotes the set of non-zero elements in $K$ ) and for every subring $R$ of $K$ which is finitely generated over $\mathbb{Z}$, the equation

$$
F(\mathbf{x})=b \text { in } \mathbf{x}=\left(x_{1}, \ldots, x_{m}\right) \in R^{m}
$$

has only finitely many solutions. This paper proves the following result: Let $F$ be a decomposable form in $m \geq 2$ variables with coefficients in $K$, which factorizes into linear factors over $K$. Let $\mathcal{L}$ denote a maximal set of pairwise linearly independent linear factors of $F$. If $F$ has the finiteness property over $K$, then $\# \mathcal{L}>2(m-1)$.
\end{abstract}

\section{INTRODUCTION}

Let $K$ be a finitely generated (but not necessarily algebraic) extension field of $\mathbb{Q}$. Let $F(\mathbf{X})=F\left(X_{1}, \ldots, X_{m}\right)$ be a form (homogeneous polynomial) in $m \geq 2$ variables with coefficients in $K$, and suppose that $F$ is decomposable (i.e., that it factorizes into linear factors over some finite extension of $K$ ). For every $b \in K^{*}$, consider the decomposable form equation

$$
F(\mathbf{x})=b \text { in } \mathbf{x}=\left(x_{1}, \ldots, x_{m}\right) \in R^{m}
$$

where $R$ is a subring of $K$ which is finitely generated over $\mathbb{Z}$. Equations of this type are of fundamental importance in the theory of Diophantine equations and have many applications in number theory. Important classes of such equations are Thue equations, when $m=2$, norm form equations, discriminant form equations and index form equations. The Thue equations are named after A. Thue [Th] who proved in the case $K=\mathbb{Q}, R=\mathbb{Z}, m=2$, that if $F$ is a binary form having at least three pairwise linearly independent linear factors in its factorization over the field of algebraic numbers, then (1.1) has only finitely many solutions. After several generalizations, Lang $[\mathrm{L}]$ finally extended Thue's result to the general case

Received by the editors December 5, 2003 and, in revised form, March 18, 2004.

2000 Mathematics Subject Classification. Primary 11D72.

The first author was supported by NSFC number 10271089. The second author was supported in part by NSA under grant number MSPF-02G-175. 
considered above (when $K$ is an arbitrary finitely generated extension of $\mathbb{Q}$ and $R$ is an arbitrary finitely generated subring of $K$ over $\mathbb{Z}$ ).

Definition. Let $K$ be a finitely generated extension field of $\mathbb{Q}$. Let $F(\mathbf{X})$ be a decomposable form in $m \geq 2$ variables with coefficients in $K$. We say that $F$ has the finiteness property over $K$ if for every $b \in K^{*}$ and for every subring $R$ of $K$ which is finitely generated over $\mathbb{Z}$, the equation

$$
F(\mathbf{x})=b \text { in } \mathbf{x}=\left(x_{1}, \ldots, x_{m}\right) \in R^{m}
$$

has only finitely many solutions.

Let $F(\mathbf{X})=F\left(X_{1}, \ldots, X_{m}\right)$ be a decomposable form with coefficients in $K$. By enlarging $K$, if necessary, we can assume that $F$ factorizes into linear factors over $K$. In 1988, Evertse and Györy [EG1] obtained a general finiteness criterion which guarantees $F$ having the finiteness property. To state their result, we first introduce some notation. Let $\mathcal{L}$ denote a maximal set of pairwise linearly independent linear factors of $F$. For every linear subspace $V$ of $K^{m}$ of dimension $\geq 1$, we write $r(V, \mathcal{L}) \geq 3$ if there are at least three linear forms in $\mathcal{L}$ which are linearly dependent on $V$, but pairwise linearly independent on $V$. A subspace $V$ of $K^{m}$ is called $\mathcal{L}$ admissible if no form in $\mathcal{L}$ vanishes identically on $V$. The result of Evertse and Györy [EG1] is as follows:

Theorem A (Evertse and Györy). Let $K$ be a finitely generated extension field of $\mathbb{Q}$. Let $F(\mathbf{X})=F\left(X_{1}, \ldots, X_{m}\right)$ be a decomposable form in $m \geq 2$ variables with coefficients in $K$, which factorizes into linear factors over $K$. Let $\mathcal{L}$ denote a maximal set of pairwise linearly independent linear factors of $F$. Then the following statements are equivalent:

(i) For every $\mathcal{L}$-admissible subspace $V$ of $K^{m}$ of dimension greater than or equal to 2 , we have $r(V, \mathcal{L}) \geq 3$,

(ii) $F$ has the finiteness property over $K$.

To use the above theorem, we need to decide whether the finiteness condition (i) in Theorem A is satisfied or not. Evertse and Györy showed that (i) holds if $\operatorname{deg} F>2(m-1)$ and any $m$ linear factors of $F$ are linearly independent. The purpose of this short paper is to provide a simple criterion for which (i) in Theorem A does not hold, hence $F$ does not have the finiteness property over $K$.

Assume that $F$ factorizes into linear factors over $K$. It is trivial to see that if $\# \mathcal{L}<m$, the condition (i) does not hold. Further, it is not very difficult to show that if $\# \mathcal{L} \leq 2(m-1)$ and any $m$ linear factors of $F$ are linearly independent, then condition (i) does not hold. In fact, in this case, if we assume that $\mathcal{L}=\left\{L_{1}, \ldots, L_{2(m-1)}\right\}$, then the linear forms $L_{1}, \ldots, L_{2(m-1)}$ define $2(m-1)$ hyperplanes $D_{1}, \ldots, D_{2(m-1)}$ in $\mathbb{P}^{m-1}(K)$. Take a point $p \in D_{1} \cap \cdots \cap D_{m-1}$ and $q \in D_{m} \cap \cdots \cap D_{2(m-1)}$. Then the line connecting $p$ and $q$ intersects the union of these hyperplanes in no more than two points. Thus (i) does not hold by taking $V$ as the vector space defined by this line. In this paper, we show that if $\# \mathcal{L} \leq 2(m-1)$ (without the condition that any $m$ linear factors of $F$ are linearly independent), then condition (i) still does not hold. Thus, we have, together with Theorem A, the following theorem.

Main Theorem. Let $K$ be a finitely generated extension field of $\mathbb{Q}$. Let $F(\mathbf{X})$ be a decomposable form in $m \geq 2$ variables with coefficients in $K$, which factorizes into 
linear factors over $K$. Let $\mathcal{L}$ denote a maximal set of pairwise linearly independent linear factors of $F$. If $F$ has the finiteness property over $K$, then $\# \mathcal{L}>2(m-1)$.

\section{Proof of the Main Theorem}

To prove the Main Theorem, we first prove the following lemma.

Lemma 2.1. For any collection of $2 n$ hyperplanes in $\mathbb{P}^{n}(K)$ there exists a line which intersects the union of these hyperplanes in no more than two points.

Proof. Let $D_{1}, \ldots, D_{2 n}$ be the given hyperplanes in $\mathbb{P}^{n}(K)$. Let $L_{i}, 1 \leq i \leq 2 n$, be the linear forms corresponding to $D_{i}$, i.e.,

$$
D_{i}=\left\{X=\left[X_{0}: \cdots X_{n}\right] ; L_{i}(X)=0\right\} .
$$

If the dimension of the space spanned by $L_{i}, 1 \leq i \leq 2 n$, does not exceed $n$, then $\bigcap_{i=1}^{2 n} D_{i} \neq \emptyset$. In this case, we can take a point $p \in \bigcap_{i=1}^{2 n} D_{i}$ and $q \notin \bigcup_{i=1}^{2 n} D_{i}$. Then the line passing $p$ and $q$ satisfies the assertion of the lemma.

So we assume that the dimension of the space spanned by $L_{i}, 1 \leq i \leq 2 n$, is $n+1$. By changing the numbering if necessary, we can assume that $L_{1}, \ldots, L_{n}$ are linearly independent, $L_{n+1}, \ldots, L_{r_{0}}$ are linearly dependent on $L_{1}, \ldots, L_{n}$, and $L_{r_{0}+1}, \ldots, L_{2 n}$ are linearly independent of $L_{1}, \ldots, L_{n}$, where $n \leq r_{0}<2 n$. Let $I_{0}=$ $\left\{1, \ldots, r_{0}\right\}$ and $I_{0}^{\prime}$ be the complement of $I_{0}$. Let $A_{0}=\{p\}=\bigcap_{i=1}^{n} D_{i}=\bigcap_{i \in I_{0}} D_{i}$ and $B_{0}=\bigcap_{i \in I_{0}^{\prime}} D_{i}$. If $B_{0} \not \subset \bigcup_{i \in I_{0}} D_{i}$. Then take a point $q \in B_{0} \backslash \bigcup_{i \in I_{0}} D_{i}$. In this case, the line passing $p$ and $q$ satisfies the assertion of the lemma. So we only need to consider the case that $B_{0} \subset \bigcup_{i \in I_{0}} D_{i}$. In this case, the product of the linear forms $L_{i}, i \in I_{0}$, vanishes identically on $B_{0}$. Since $B_{0}$ is the intersection of some hyperplanes, it is a subspace of $\mathbb{P}^{n}(K)$. So its polynomial ring is an integral domain. Hence one of the linear forms in $\left\{L_{i}, i \in I_{0}\right\}$, say $L_{i_{0}}, i_{0} \in I_{0}$, must vanish identically on $B_{0}$. Therefore, $B_{0} \subset D_{i_{0}}$ for some $i_{0} \in I_{0}$. Consider $I_{0} \backslash\left\{i_{0}\right\}$. We claim that there exist $n-1$ linearly independent linear forms among $L_{i}, i \in I_{0} \backslash\left\{i_{0}\right\}$, such that $L_{i_{0}}$ is linearly independent of them. In fact, if $1 \leq i_{0} \leq n$, then $L_{i}, 1 \leq$ $i \leq n, i \neq i_{0}$, are the desired linear forms. If $i_{0}>n$, then $L_{i_{0}}$ is linearly dependent on $\left\{L_{1}, \ldots, L_{n}\right\}$. The claim follows from the following simple linear algebra fact that given vectors $\beta, \alpha_{1}, \ldots, \alpha_{k}$, such that $\alpha_{1}, \ldots, \alpha_{k}$ are linearly independent, and $\beta, \alpha_{1}, \ldots, \alpha_{k}$ are linearly dependent, then there exist $i_{1}, \ldots, i_{k-1}$ among $1, \ldots, k$ such that $\beta, \alpha_{i_{1}}, \ldots, \alpha_{i_{k-1}}$ are linearly independent. Hence the claim holds. By the claim, without loss of generality, we assume that $L_{i_{0}}$ is linearly independent of $L_{1}, \ldots, L_{n-1}$. Let $I_{1}$ be the index set such that each $L_{j}, j \in I_{1}$, is linearly dependent on $L_{1}, \ldots, L_{n-1}$, and let $I_{1}^{\prime}$ be the complement of $I_{1}$. Then $I_{0}^{\prime} \subset I_{1}^{\prime}$ and $i_{0} \in I_{1}^{\prime}$. Let $B_{1}=\bigcap_{i \in I_{1}^{\prime}} D_{i}$. Then

$$
\begin{aligned}
B_{1} & =\bigcap_{i \in I_{1}^{\prime}} D_{i}=\bigcap_{i \in I_{0}^{\prime}} D_{i} \cap D_{i_{0}} \bigcap_{i \in I_{1}^{\prime} \backslash\left(\left\{i_{0}\right\} \cup I_{0}^{\prime}\right)} D_{i} \\
& =B_{0} \cap D_{i_{0}} \bigcap_{i \in I_{1}^{\prime} \backslash\left(\left\{i_{0}\right\} \cup I_{0}^{\prime}\right)} D_{i} \\
& =B_{0} \bigcap_{i \in I_{1}^{\prime} \backslash\left(\left\{i_{0}\right\} \cup I_{0}^{\prime}\right)} D_{i} .
\end{aligned}
$$


Hence,

$$
\begin{aligned}
\operatorname{dim} B_{1} \geq \operatorname{dim} B_{0}-\left(\# I_{1}^{\prime}-\# I_{0}^{\prime}-1\right) & \geq r_{0}-n-\# I_{1}^{\prime}+\left(2 n-r_{0}\right)+1 \\
& =n+1-\# I_{1}^{\prime}=\# I_{1}-(n-1) \geq 0 .
\end{aligned}
$$

It follows that $B_{1} \neq \emptyset$. We set $A_{1}=\bigcap_{i=1}^{n-1} D_{i}$. Then $\operatorname{dim} A_{1}=1$. Hence $A_{1} \backslash \bigcup_{i \in I_{1}^{\prime}} D_{i} \neq \emptyset$. If $B_{1} \not \subset \bigcup_{i \in I_{1}} D_{i}$, then the line passing through an arbitrary pair of points $p \in A_{1} \backslash \bigcup_{i \in I_{1}^{\prime}} D_{i}, q \in B_{1} \backslash \bigcup_{i \in I_{1}} D_{i}$ satisfies the assertion of the lemma. The remaining case is $B_{1} \subset \bigcup_{i \in I_{1}} D_{i}$. Then, in this case, $B_{1} \subset D_{j}$ for some $1 \leq j \in I_{1}$. Proceeding just as above, we construct sets $A_{2}$ and $B_{2}$, etc. If this iterative process continues to the $k$-th step $(k<n)$, then there are defined numbers $r_{k} \leq r_{k-1} \leq \cdots \leq r_{0}$ and sets $A_{k}$ and $B_{k}$, such that (in the corresponding enumeration):

(1) $A_{k}=\bigcap_{i \in I_{k}} D_{i}, A_{k} \not \subset \bigcup_{i \in I_{k}^{\prime}} D_{i}$, and $\operatorname{dim} A_{k}=k$;

(2) $B_{k}=\bigcap_{i \in I_{k}^{\prime}} D_{i}, \operatorname{dim} B_{k} \geq \# I_{k}-(n-k) \geq 0$.

Now, if $B_{k} \not \subset \bigcup_{i \in I_{k}} D_{i}$, then the line passing through an arbitrary pair of points $p \in A_{k} \backslash \bigcup_{i \in I_{k}^{\prime}} D_{i}, q \in B_{k} \backslash \bigcup_{i \in I_{k}} D_{i}$ satisfies the assertion of the lemma. In this case, the process stops with the line we want. Otherwise, $B_{k} \subset \bigcup_{i \in I_{k}} D_{i}$. In this case, we continue the iterative procedure: the $(k+1)$-st step is executed analogously to the first. If the process does not end with the construction of the line sought by the $(n-1)$-st step, then at the $(n-1)$-step we get $A_{n-1}=D_{1}, B_{n-1}=$ $\bigcap_{i=2}^{2 n} D_{i}, \operatorname{dim} B_{n-1} \geq 0$, and since by assumption $\bigcap_{i=1}^{2 n} D_{i}=\emptyset$, one has $D_{1} \not \supset B_{n-1}$. Obviously $A_{n-1} \not \subset \bigcup_{i=2}^{2 n} D_{i}$ and consequently the line passing through an arbitrary pair of points $q \in A_{n-1} \backslash \bigcup_{i=2}^{2 n} D_{i}, q \in B_{n-1} \backslash D_{1}$ is the one we sought. The lemma is thus proved.

We are now ready to prove the Main Theorem.

Proof. Let $F(\mathbf{X})=F\left(X_{1}, \ldots, X_{m}\right)$ be the decomposable form given in the Main Theorem. Let $\mathcal{L}=\left\{L_{1}, \ldots, L_{q}\right\}$ denote a maximal set of pairwise linearly independent linear factors of $F$. Denote by $D_{1}, \ldots, D_{q}$ the hyperplanes in $\mathbb{P}^{m-1}(K)$ defined by the linear forms $L_{1}, \ldots, L_{q}$. Suppose the Main Theorem is not true, i.e., $\# \mathcal{L} \leq 2(m-1)$. By Lemma 2.2, there exists a line which intersects the union of $D_{1}, \ldots, D_{q}$ in no more than two points. Let $V$ be the subspace of $K^{m}$, determined by this line. Then $V$ is $\mathcal{L}$-admissible and $r(V, \mathcal{L})<3$. By Theorem A, $F$ does not have the finiteness property. This contradicts the assumption of the Main Theorem. Therefore the Main Theorem is proved.

\section{ACKNOWLEDGMENT}

The second author wishes to thank the Department of Mathematics, Tongji University, P.R. China, for kind hospitality during which part of the work on this paper took place.

\section{REFERENCES}

[EG1] Evertse, J.H. and Györy, K., Finiteness criteria for decomposable form equations, Acta Arith. 50 (1988), 357-379. MR0961695 (90a:11041)

[EG2] Evertse, J.H. and Györy, K., Decomposable form equations. In: New advances in transcendental theory (ed. by A. Baker), 175-202. Cambridge Univ. Press, Cambridge 1988. MR0971999 (89i:11042) 
[EG3] Evertse, J.H. and Györy, K., Some applications of decomposable equations to resultant equations, Colloq. Math. 65 (1993), 267-275. MF 1240172 (94k:11036)

[G] Györy, K., On the distribution of solutions of decomposable form equations, Number Theory in Progress, Walter de Gruyter, Berlin, New York 1 (1999), 237-265. MR1689508 (2000e:11036)

[GR] Györy, K. and Ru, M., Integer solutions of a sequence of decomposable form inequalities, Acta Arith. (1998). MR.1655981 (2000a:11044)

[K] Kiernan, P., Hyperbolic submanifolds of complex projective space, Proc. Amer. Math. Soc. 3 (1968), 603-606. MR0245828 (39:7134)

[L] Lang, S., Fundamentals of Diophantine Geometry, Springer, Berlin Heidelberg New York, 1983. MR0715605 (85j:11005)

[RV] Ru, M. and Vojta, P., Schmidt's subspace theorem with moving targets, Invent. Math. 127 (1997), 51-65. MF1423025 (97g:11076)

[RW] Ru, M. and Wong, P.M., Integral points of $\mathbb{P}^{n}-\{2 n+1$ hyperplanes in general position $\}$, Invent. Math. 106 (1991), 196-216. MR1123379 (93f:11056)

[Sch1] Schmidt, W.M., Norm form equations, Ann. of Math. (2)96 (1972), 526-551. MR0314761 $(47: 3313)$

[Sch2] Schmidt, W.M., Diophantine approximations, Lect. Notes Math. Vol 785, Springer, Berlin Heidelberg New York, 1980. MR0568710 (81j:10038)

[Sn] Snurnitsyn, V.E., The complement of $2 n$ hyperplanes is not hyperbolic, Mat. Zametki 40 (1986), 455-459. MR0873474 (88e:32037)

[Th] Thue, T., Über Annäherungswerte algebraischer Zahlen, J. Reine Angew. Math., 135 (1909), 284-305.

[V] Vojta, P., Diophantine Approximations and Value Distribution Theory, Lect. Notes Math. Vol. 1239, Springer, Berlin Heidelberg New York, 1987. MR0883451 (91k:11049)

Department of Mathematics, Tongu University, Shanghai, People's Republic of CHINA

E-mail address: zzzhhc@tongji.edu.cn

Department of Mathematics, University of Houston, Houston, Texas 77204

E-mail address: minru@math.uh.edu 